\title{
Pulmonary function testing is safe in patients with thoracic aortic aneurysms
}

\author{
To the Editor:
}

Thoracic aortic aneurysms (TAA) occur in up to 16 patients per 100000 of the population and are increasingly amenable to surgical correction [1]. However, low lung function is associated with poor post-operative outcomes, and a pre-operative assessment of pulmonary function is therefore important for prognostication [2].

The American Association of Respiratory Care (AARC) 1996 Spirometry Clinical Practice Guidelines listed thoracic, abdominal and cerebral aneurysms as relative contraindications to forced respiratory manoeuvres due to the perceived danger of rupture from increased thoracic pressure [3]. Despite this caveat, there is little evidence for this and a recent study found lung function testing is safe in the presence of abdominal aneurysms $<6 \mathrm{~cm}$ [4]. The AARC guideline has now been retired, however, little is known about the risk of rupture in TAA following forced expiratory manoeuvres and hence the AARC contraindications are still often referred to [5].

To assess the true risk of rupture following TAA surgery and the effect of spirometric measurement, we reviewed the outcomes of all patients who had undergone TAA surgery between 2010 and 2017. Our institute is a tertiary cardiothoracic centre providing regional elective, urgent and emergency surgical management of TAA, where it is routine practice to obtain pulmonary function testing for patients undergoing surgery where possible. Spirometry is performed by an accredited respiratory physiologist according to American Thoracic Society/European Respiratory Society guidelines [6]. The local protocol is uniform across indications and all patients are instructed to perform at least three repeatable forced expiratory manoeuvres.

Baseline demographics, lung function, TAA characteristics and post-operative outcomes, including 30-day mortality and length of stay (LOS), were recorded from our institutional surgical and pulmonary function laboratory databases. We then investigated associations between pre-operative spirometry and mortality, as well as operative outcomes including length of stay and 30-day mortality. We also investigated whether any subjects with planned elective TAA required unplanned urgent/emergency operative intervention in the time-period following spirometry. Finally, hospital records of medical emergency team/cardiac arrest alerts were examined. All predicted lung function values are presented as calculated from the Global Lung Initiative reference equations [7]. Data are presented as median (interquartile range) or $\mathrm{n}(\%)$.

Between January 2011 and March 2017, 519 patients underwent open surgical management of TAA at our institute (427 (82.2\%) out of 519 elective, 69 (13.3\%) out of 519 urgent and 23 (4.4\%) out of 519 emergency). Median aneurysm size was $5.6(5.0-6.3) \mathrm{cm}(5.5(5.0-6.0) \mathrm{cm}, 6.5(5.0-8.0) \mathrm{cm}$ and $5.0(5.0-8.0) \mathrm{cm}$ for each surgical group, respectively). Baseline characteristics are presented in table 1 . Spirometry was performed in 426 (82.1\%) out of 519 with an average forced expiratory time of 9 (7-12) s, and American Thoracic Society/ European Respiratory Society acceptability criteria were met in $325(82.2 \%)$ out of 426 . Average forced expiratory volume in $1 \mathrm{~s}$ and forced vital capacity were $88.3(75.8-100.0) \%$ pred and $95.0(83.8-105.6) \%$ pred, respectively, airway obstruction was present in 145 (33.9\%) out of 426 cases and time to operation was 35 (7.2-57.4) days. Post-operative LOS was inversely related to forced expiratory volume in $1 \mathrm{~s}(\mathrm{r}=-0.2$ $\mathrm{p}<0.01)$. There were no events of sudden deterioration requiring the medical emergency team or cardiac arrest team in the pulmonary function lab event records or amongst the hospital medical emergency team records.

In the elective group, $387(90.1 \%)$ out of 427 patients had pre-operative spirometry and there was no difference in 30-day post-operative mortality or LOS compared to the remainder (5.4\% versus $7.5 \%$, $\mathrm{p}=0.32$; and 16.2 days versus $16.3, \mathrm{p}=0.96)$. Similarly, in the urgent group, the $39(55.1 \%)$ patients with

@ERSpublications

Spirometry is safe as part of pre-operative work-up in patients with thoracic aortic aneurysms http://ow.ly/uwSe301J6Qn

Cite this article as: Frost F, Peat R, McWean J, et al. Pulmonary function testing is safe in patients with thoracic aortic aneurysms. Eur Respir J 2018; 52: 1800928 [https://doi.org/10.1183/13993003.00928-2018]. 


\begin{tabular}{|c|c|c|c|c|}
\hline & All & Elective $^{\#}$ & Urgent $^{\pi}$ & Emergency \\
\hline Subjects & $519(100)$ & 427 (82.2) & 69 (13.3) & $23(4.4)$ \\
\hline Age years & $66.9(54.7-73.4)$ & 66.9 (55.3-73.5) & $67.6(52.0-74.7)$ & $62.2(46.6-73.2)$ \\
\hline Female & $188(36.2)$ & $161(37.7)$ & $25(36.2)$ & $2(8.7)$ \\
\hline FEV1 L & $2.5(1.9-3.1)$ & 2.5 (1.9-3.2) & $2.1(1.7-2.7)$ & N/A \\
\hline FEV $1 \%$ pred & $88.4(75.8-100.0)$ & $88.9(76.6-100.6)$ & 80.5 (69.5-93.3) & N/A \\
\hline FVC L & $3.3(2.6-4.1)$ & $3.3(2.6-4.2)$ & $2.9(2.2-3.7)$ & $\mathrm{N} / \mathrm{A}$ \\
\hline FVC \% pred & $95.0(83.8-105.6)$ & 95.8 (85.1-107.5) & $87.3(79.8-98.6)$ & N/A \\
\hline FET $s$ & $9(7-12)$ & $9(7-12)$ & $10(7-13)$ & N/A \\
\hline Size of TAA cm & $5.6(5.0-6.3)$ & $5.5(5.0-6.0)$ & $6.5(5.0-8.0)$ & $5.0(5.0-8.0)$ \\
\hline \multicolumn{5}{|c|}{$\begin{array}{l}\text { Data are presented as } \mathrm{n}(\%) \text { or median (interquartile range). FEV1: forced expiratory volume in } 1 \mathrm{~s} \text {; } \\
\text { FVC: forced vital capacity; FET: forced expiratory time; N/A: no spirometry performed prior to emergency } \\
\text { surgery; TAA: thoracic aortic aneurysm. }{ }^{\#}: 387 \text { out of } 427 \text { underwent spirometry; }{ }^{\text {ๆ }}: 39 \text { out of } 69 \text { underwent } \\
\text { spirometry. }\end{array}$} \\
\hline
\end{tabular}

pre-operative spirometry had no difference in 30-day mortality or LOS compared to those who did not have spirometry (four (10.\%) out of 38 versus two (6.4\%) out of $31, \mathrm{p}=0.769$; and 18.2 days versus 17.4 days, $\mathrm{p}=0.97$ ). No patient in the emergency group (30-day mortality $8.7 \%$; LOS 13.8 days) had spirometry immediately prior to surgery.

Of those with TAA $>6 \mathrm{~cm}, 175$ (81\%) out of 217 underwent spirometry; there was no difference in 30-day mortality compared to the remainder (16 (9.1\%) out of 175 versus eight (19.5\%) out of 42 , $\mathrm{p}=0.6$ respectively).

We have shown that the forced respiratory manoeuvre necessary for spirometric measurement was not associated with adverse outcomes in patients undergoing the surgical management of TAA and there was no increase in mortality, LOS or the need for unplanned urgent/emergency surgery in patients having undergone spirometry. Our findings were similar across all sizes of TAA. However, we found lung function is an important predictor of post-operative LOS, underlining the value of its assessment.

Aneurysms were first listed as a relative contraindication due to the theoretical risk of rupture and hence testing may understandably cause concern for patients, clinicians and physiologists alike [3]. However, to our knowledge there are no reported cases of aneurysmal rupture following pulmonary function testing.

Pre-operative spirometry is routinely performed in the work-up for thoracic aortic aneurysm surgery at our centre, other centres and also in clinical trials where it has been well validated as a predictor of post-operative outcomes [2, 8, 9]. More recently, the safety of spirometry in abdominal aortic aneurysms has been demonstrated [4]. To our knowledge, ours is the largest study to date investigating the safety of spirometry in TAA. Other studies have investigated spirometry in terms of risk-stratification for aortic surgery without reports of any significant adverse events, further supporting its utility [10]. In conclusion, we found no evidence of acute rupture of TAA or poorer outcomes in patients with TAA following spirometry.

Freddy Frost $\oplus^{1}$, Rob Peat ${ }^{2}$, Joan McWean ${ }^{2}$, Matt Shaw ${ }^{3}$, Mark Field ${ }^{4}$, Dilip Nazareth ${ }^{1}$ and Martin Walshaw ${ }^{1}$ ${ }^{1}$ Respiratory Dept, Liverpool Heart and Chest Hospital, Liverpool, UK. ${ }^{2}$ Pulmonary Function Dept, Liverpool Heart and Chest Hospital, Liverpool, UK. ${ }^{3}$ Research Dept, Liverpool Heart and Chest Hospital, Liverpool, UK. ${ }^{4}$ Cardiothoracic Surgery Dept, Liverpool Heart and Chest Hospital, Liverpool, UK.

Correspondence: Freddy Frost, Respiratory Dept, Liverpool Heart and Chest Hospital, Thomas Drive, Liverpool L14 3PE, UK. E-mail: freddy.frost@lhch.nhs.uk

Received: May 172018 | Accepted after revision: Aug 282018

Conflict of interest: None declared.

\section{References}

1 Olsson C, Thelin S, Stahle E, et al. Thoracic aortic aneurysm and dissection: increasing prevalence and improved outcomes reported in a nationwide population-based study of more than 14,000 cases from 1987 to 2002 . Circulation 2006; 114: 2611-2618.

2 Girardi LN, Lau C, Munjal M, et al. Impact of preoperative pulmonary function on outcomes after open repair of descending and thoracoabdominal aortic aneurysms. J Thorac Cardiovasc Surg 2017; 153: S22-S29.

3 American Association for Respiratory Care. AARC Clinical Practice Guidelines. Spirometry. 1996 update. Respir Care 1996; 41: 629-636. 
4 Zagami D, Wilson J, Bodger A, et al. Respiratory function testing is safe in patients with abdominal aortic aneurysms. Vasc Endovascular Surg 2014; 48: 522-523.

5 Cooper BG. An update on contraindications for lung function testing. Thorax 2011; 66: 714-723.

Miller MR, Hankinson J, Brusasco V, et al. Standardisation of spirometry. Eur Respir J 2005; 26: 319-338.

Quanjer PH, Hall GL, Stanojevic S, et al. Age- and height-based prediction bias in spirometry reference equations. Eur Respir J 2012; 40: 190-197.

8 Meijer CA, Kokje VB, van Tongeren RB, et al. An association between chronic obstructive pulmonary disease and abdominal aortic aneurysm beyond smoking: results from a case-control study. Eur J Vasc Endovasc Surg 2012; 44: 153-157.

9 Ohrlander T, Nessvi S, Gottsater A, et al. Influence of preoperative medical assessment prior to elective endovascular aneurysm repair for abdominal aortic aneurysm. Int Angiol 2012; 31: 368-375.

10 Ivanov A, Yossef J, Tailon J, et al. Do pulmonary function tests improve risk stratification before cardiothoracic surgery? J Thorac and Cardiovasc Surg; 151: 1183-1189. 